\title{
The Problem of Energy Transfer in the Crab Nebula
}

\author{
G. Machabeli, G. Mchedlishvili, D. Shapakidze \\ Theoretical Dept., Abastumani Astrophysical Observatory of Georgian \\ Academy of Sciences, Al. Kazbegi ave. 2a, 380060 Tbilisi, Republic of \\ Georgia
}

\begin{abstract}
The possibility of Langmuir wave generation in electronpositron plasma is considered. As a result of induced scattering, these waves find themselves in the superluminal region. Thereby such waves can transfer energy to the Crab Nebula essentially without loss, where the remaining plasma creates condition of collapse of Langmuir waves. As a result, the tail of the $e^{ \pm}$distribution function stretchs out to Lorentz factors of the order of $\gamma_{ \pm} \leq 10^{9}$. Via synchrotron emission, particles of these energies can radiate $X$ and $\gamma$-radiation.
\end{abstract}

\section{Introduction}

First of all, two notes: 1) we believe that the only source of the Nebula energy is PSR $0531+21 ; 2$ ) it is assumed that the nonthermal radiation process of the Nebula is synchrotron emission.

There are two main problems: i) how is the energy transported from the pulsar to the Nebula in such a way, that it does not reveal itself till $\sim 3 \times 10^{17} \mathrm{~cm}$ ? ii) what is the acceleration mechanism of the particles which give rise to high frequency radiation? We will enlarge only on the second problem and offer schematically our own plasma model on the particle acceleration.

\section{Strong Langmuir Turbulence}

Ruderman and Sutherland (1975) suggested the generation of Langmuir oscillations (l-wave) by a beam of primary particles with Lorentz factor $\gamma_{b}$. However, detailed consideration showed that kinetic instability does not develop (Egorenkov, Lominadze, Mamradze 1983); it turns out that $\gamma_{b}$ is too high. Cheng and Ruderman (1977) suggested substituting an electron beam for the beam of ions. The Lorentz factor then decreases by a factor $\left(m_{e} / m_{i}\right)$ and the distribution function has the form shown in figure 1. It is obvious that there are many more particles upon which the waves can damp than just the ions, so excitation is out of the question. Electrons with big $\gamma$-factors reach distances $R \sim 3 \times 10^{17} \mathrm{~cm}$. The power in curvature radiation is

$$
\frac{d \varepsilon}{d t}=-\frac{2}{3} \gamma^{4} \frac{e^{2} c}{r_{B}^{2}}\left(\frac{m_{e}}{m_{i}}\right)^{2}
$$




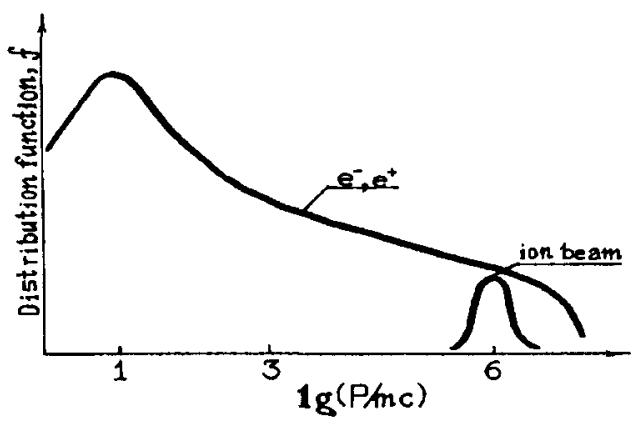

Figure 1. The distribution function of the pulsar magnetosphere particles within the light cylinder.

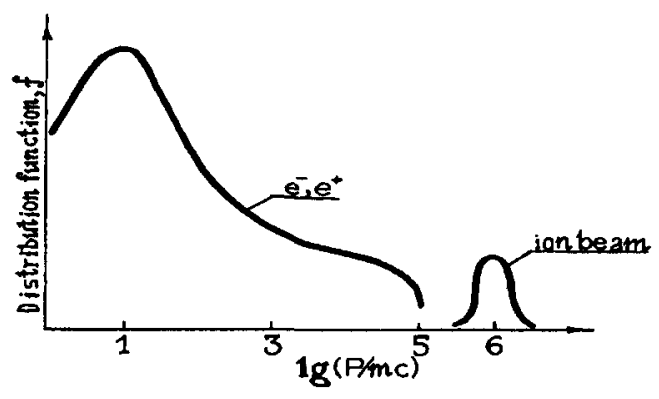

Figure 2. The distribution function of magnetospheric particles beyond the light cylinder.

and this limits their Lorentz factors to values $\gamma_{ \pm} \leq 10^{5}$. The ion beam reaches radius $R$ essentially without energy loss, so beyond the light cylinder the distribution function acquires the form shown in figure 2. Moreover the ion beam broadens, creating thereby conditions for Langmuir wave generation. The spectrum of Langmuir potential waves is shown in figure 3 . The excitation is possible in area I only, where the instability growth rate is

$$
\Gamma \simeq \frac{m_{e}}{m_{i}} \frac{n_{b}}{n_{p}} \dot{\gamma}_{b}^{3} \gamma_{p} \frac{\partial f}{\partial p_{\|}} k_{\|} c .
$$

Because of induced scattering by the $e^{ \pm}$-plasma particles (see figure 4), wave energy is transmitted to the superluminal area, where waves do not interact with the particles, and a so-called "plasmon condensate" is created.

While propagating to the periphery, the $l$-wave acquires some transverse component and the spectrum has the following form:

$$
\omega=\frac{\omega_{p}}{\gamma^{1 / 2}}\left\{\left(1+\frac{1}{2} \frac{k_{\|}^{2} c^{2}}{\omega_{p}^{2}}\right)+\frac{k_{\perp}^{2} c^{2}}{\omega_{p}^{2}} \frac{\omega_{B}^{2}}{\omega_{p}^{2}}\right\}
$$




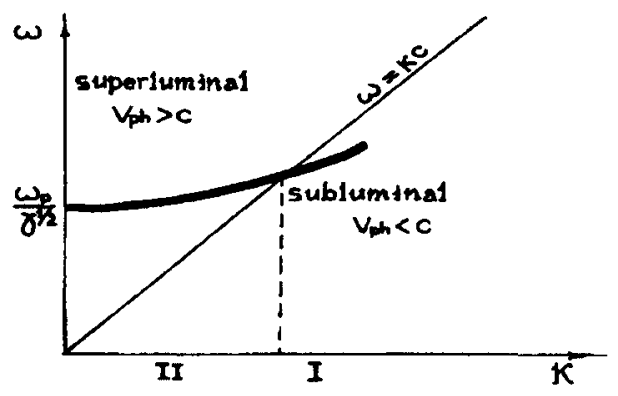

Figure 3. The spectrum of the potential Langmuir waves: I - the subluminal area of the spectrum, II - the superluminal area of spectrum.

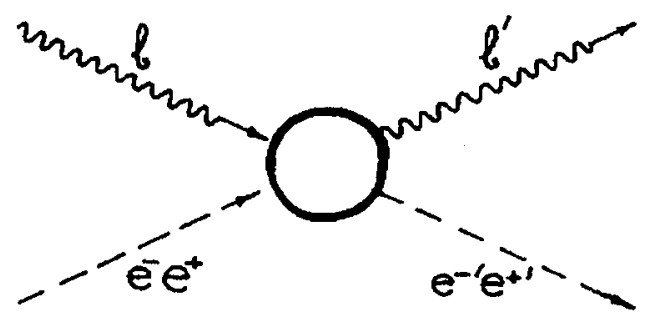

Figure 4. The induced scattering upon the $e^{ \pm}$-plasma particles.

The Langmuir wave becomes 3-dimensional and the collapse begins (Zakharov 1973). This means that, via their pressure gradient, the waves in the "condensate" expel particles, thus forming cavities or "density pits".

We suppose, that in the Nebula some ions remain and the condition $T_{\varepsilon} \gg T_{i}$ is met. We can set up a system of nonlinear equations, where the nonlinear term is defined by the decay process

$$
\begin{gathered}
l \rightarrow l+s \quad(\mathrm{~s}=\text { ion sound wave) } \\
\left(i \frac{\partial}{\partial t}+\frac{1}{2} \frac{c^{2}}{\omega_{p}^{2}} \frac{\partial^{2}}{\partial z^{2}}\right) E_{z}+\frac{\omega_{B e}^{2}}{\omega_{p e}^{2}} \frac{c^{2}}{\omega_{p}^{2}}\left(\frac{\partial^{2}}{\partial x^{2}}+\frac{\partial^{2}}{\partial y^{2}}\right) E_{z} \simeq \frac{\omega_{B e}}{\omega_{p e}} \frac{\partial^{2}}{\partial z^{2}}(\delta n E(z, t)), \\
\left(\frac{\partial^{2}}{\partial t^{2}}-\frac{T_{e}}{m_{i}} \frac{\partial^{2}}{\partial z^{2}}\right) \delta n=\frac{1}{8 \pi} \frac{1}{m_{i}} \frac{\partial^{2}}{\partial z^{2}}|E|^{2}
\end{gathered}
$$

and the solution gives:

$$
E \sim \frac{1}{\tau_{0}-\tau}, \quad \tau_{0}=\omega_{p} \frac{E_{0}^{2}}{8 \pi n T_{0}} t .
$$




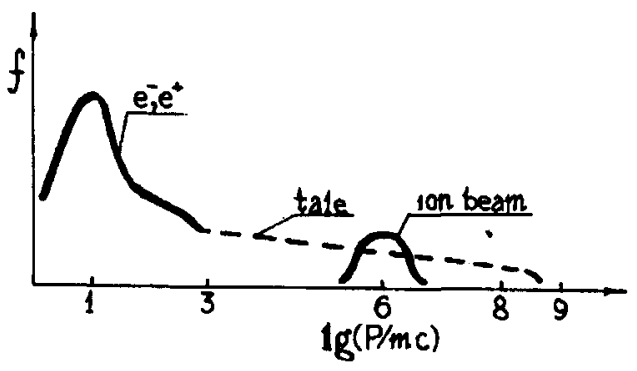

Figure 5. The elongation of the distribution function tail.

The collapse proceeds with increasing velocity. The wavelength decreases as well as the phase velocity, and the wave energy is pumped into short wavelengths. The fastest particles will reach resonance with the wave first and are accelerated as they Landau-damp the wave. Thus we expect growth of the tail of the distribution function up to Lorentz factors of the order of $\gamma_{ \pm} \leq 10^{9}$. Such Lorentz factors could explain the hard $\gamma$-radiation of the Crab Nebula.

\section{Conclusions}

Let us summarize our results: we will schematically present the succession of the processes which lead to energy transfer from the pulsar to the Nebula, and transformation of this energy to $X$ and $\gamma$-radiation.

Ion beam $\rightarrow$ generation of Langmuir wave $\rightarrow l+e^{ \pm} \rightarrow l^{\prime}+e^{ \pm \prime}$ and condensate formation $\longrightarrow 3$-dimensional spectrum and the presence of ions $\longrightarrow$ collapse of Langmuir waves $\longrightarrow$ leads to $\lambda$ decrease, Landau damping $\longrightarrow$ particle tail stretching $\longrightarrow X, \gamma-$ ray emission.

\section{References}

Cheng A. F. and Ruderman M. 1977, ApJ, 212, 800

Egorenkov V. D., Lominadze D. J. and Mamradze P.G. 1983, Astrofizika, 19, 753

Ruderman M. and Sutherland P. G. 1975, ApJ, 196, 51

Zakharov V. E. 1972, Sov. Phys., 35, 908 